\title{
Diferenciales de ingreso por el color de la piel y desigualdad de oportunidades en Colombia*
}

\author{
Recibido: 30 de abril de 2016- Aceptado: 24 de abril de 2017
}

Doi: dx.doi.org/10.12804/revistas.urosario.edu.co/economia/a.6151

Carlos Augusto Viáfara López+

Resumen

El objetivo de este artículo es tratar de separar los efectos asociados con la transmisión intergeneracional de la escolaridad de los efectos de la discriminación racial en los diferenciales de ingreso, según el color de la piel en Colombia. Los datos corresponden a la Encuesta Nacional de Hogares (ENH) de Diciembre del 2000. Se estimaron ecuaciones de ingresos por medio de Mínimos Cuadrados Ordinarios en dos Etapas (MC2E) y se realizaron microsimulaciones. Los resultados muestran que una proporción no deleznable de los diferenciales de ingreso están asociados con la transmisión intergeneracional de las desigualdades por el color de la piel en Colombia.

Palabras clave: diferenciales de ingreso, movilidad intergeneracional, discriminación en el mercado laboral.

Clasificación JEL: J15, J31, J62, J71

* Este artículo se deriva del proyecto de investigación "Desigualdad en la distribución del ingreso en Colombia: un análisis a partir de la Regresión Cuantílica" con código Colciencias 1106452-21309. Fue realizado en el grupo de investigación, Crecimiento Económico y Mercado Laboral de la Universidad del Valle. Agradezco la financiación de la Universidad del Valle y de Colciencias. Agradezco los comentarios de un evaluador anónimo que sirvieron para jerarquizar este artículo. Además, estoy en deuda con el economista Alexander Banguera Obregón por su invaluable colaboración durante el desarrollo de esta investigación.

+ Profesor asociado del Departamento de Economía, Facultad de Ciencias Sociales y Económicas, Universidad del Valle, Cali, Colombia. Correspondencia: calle 13 \# 100-00, edificio 387, oficina 3036, teléfono 3212322, fax 3315200.Correo electrónico: carlos.viafara@ correounivalle.edu.co

Para citar este artículo: Viáfara-López, C. (2017). Diferenciales de ingreso por el color de la piel y desigualdad de oportunidades en Colombia. Revista de Economía del Rosario, 20(1), 97-126. Doi: dx.doi.org/10.12804/revistas.urosario.edu.co/economia/a.6151 


\title{
Wage Differentials by Skin Color and Inequality of Opportunities in Colombia
}

\begin{abstract}
This article attempts to isolate the effects of intergenerational transmission of schooling from the racial discrimination effects on income differentials according to skin color in Colombia. For this proposal, it is used the sample of the ENH of December of 2000. Income equations were estimated by Two Stage Least Square Model (2sLS) and microsimulations were made. The results reveal that a non-negligible part of the component of income differentials is explained by the intergenerational transmission of inequalities due to skin color in Colombia.
\end{abstract}

Keywords: Racial Discrimination; Wage Differentials; Intergenerational Mobility. JEL Classification: J15, J31, J62, J71

\section{Diferenciais de Ingresso pela cor da pele e desigualdade de oportunidades na Colômbia}

\author{
Resumo
}

O objetivo deste artigo é tentar separar os efeitos associados à transmissão intergeracional da escolaridade dos efeitos da discriminação racial nos diferenciais de ingresso segundo a cor da pele na Colômbia. Os dados correspondem ao Inquérito Nacional de Lares de Dezembro do ano 2000. Estimaram-se equações de ingressos através de Mínimos Quadrados Ordinários em duas Etapas (MC2E) e se realizaram microsimulações. Os resultados mostram que uma proporção não depreciável dos diferenciais de ingresso está associados à transmissão intergeracional das desigualdade pela caro da pele na Colômbia.

Palavras-chave: diferenciais de ingresso, mobilidade intergeracional, discriminação no mercado laboral

Classificação JEL: J15; J31; J62; J71.

\section{Introducción}

La equidad está referida a un concepto de justicia social en el proceso que conduce a los resultados tales como el ingreso, como opuesto a la igualdad de resultados o de ingreso per se (Sen, 1992). En consecuencia, la igualdad de oportunidades adopta la idea de que los individuos deberían ser responsables de sus elecciones, en cuanto pueden ser redimidos de los efectos de las circunstancias y escogen los planes de vida que cada cual estime conveniente (Roemer, 1998). 
En sociedades como la latinoamericana, fundadas por instituciones de desigualdad de larga duración (Engerman \& Sokoloff, 1997), los resultados no solo se determinarían por el esfuerzo o logro individual, principio fundamental de toda sociedad meritocrática, sino que también estarían relacionados con las características circunstanciales que enfrentan los individuos.

En ese aspecto de las características circunstanciales, el color de la piel pareciera cumplir un rol importante en la transmisión intergeneracional de las desigualdades, debido a la innegable asociación entre condición étnicoracial, pobreza y marginalidad en Colombia y en otros países de América Latina (Flórez, Medina y \& Urrea, 2001; Urrea \& Viáfara, 2007). No es coincidencia que hasta el momento la mayoría de los estudios sobre desigualdad de oportunidades en la región se hayan realizado sobre Brasil, el país de mayor población negra de América Latina (Soares, 2000; Bourguignon et al., 2003, Campante et al., 2004, Leite, 2005).

Ahora bien, en Colombia ha habido una investigación razonable de la transmisión intergeneracional de la educación (Nina \& Grillo, 2000; Behrman, Gaviria \& Székely, 2001; Gaviria, 2002; Cartagena, 2004; Tenjo, 2004; Viáfara 2005, 2006; Viáfara \& Urrea 2006; Bonilla, 2010; Viáfara, Estacio \& González, 2010; Galvis-Aponte \& Meisel-roca 2015), además, y quizás lo más importante para efectos de este artículo, se han intentado auscultar los factores asociados con la transmisión intergeneracional en los resultados económicos (Nuñez et al., 2006; Ferreira \& Meléndez 2010; Ruiz, 2011). Más allá de la importancia de estas investigaciones para entender la desigualdad de oportunidades en Colombia, llama la atención que hasta ahora no se conozca la incidencia de la desigualdad de oportunidades en los diferenciales salariales entre grupos sociales determinados por el color de la piel ${ }^{1}$.

En este sentido, las preguntas que intenta resolver este artículo son: ¿Existen desigualdades de ingresos en personas diferenciadas por el color de la piel en Colombia? Si lo anterior es afirmativo, ¿En qué grado los diferenciales de ingresos observados se explican por la desigualdad de oportunidades que ellos heredan de sus padres y que resulta en alguna heterogeneidad en los esfuerzos? o, por el contrario, ¿ son el resultado de los retornos a esos esfuerzos?

La estrategia metodológica involucra, en primer lugar, la estimación de un modelo de raza- intercepto en mínimos cuadrados ordinarios (MCO). Luego se estima un modelo de raza- intercepto en mínimos cuadrados en dos etapas

1 Cabe mencionar que según Andrews (2016), Colombia era el cuarto país con mayor concentración de población afrodescendientes en América Latina con aproximadamente 4312000 habitantes en la ronda de censos de 2000, después de Brasil, Venezuela y República Dominicana. 
(MC2E), con el objetivo de corregir el posible sesgo de estimar el modelo sin instrumentos para la escolaridad individual debido a su correlación con la educación de los padres.

Posteriormente, se estiman modelos de intercepto específico por (MCO y MC2E) para las personas que se autorreconocieron como negros, mulatos, mestizos y blancos; esta metodología permite instrumentar la escolaridad con los antecedentes familiares. Más adelante, se aplica el método de descomposición de la brecha salarial (adaptación de la metodología Blinder-Oaxaca), microsimulasiones con base en la metodología de Bourguignon et al., (2003) y Leite (2005), para estimar las ganancias potenciales de los Negros bajo el supuesto de que son pagados como los otros grupos. Además, las ganancias potenciales de los negros con la hipótesis de que tienen las mismas circunstancias (educación de los padres) de los otros grupos.

El método permite auscultar el proceso de desventajas acumulativas para los trabajadores negros. En primer lugar, y derivado de sus antecedentes familiares más empobrecidos, tienen menos posibilidades de hacer carreras educativas largas y de calidad, lo cual determina mayormente salarios más bajos en el mercado laboral. Se podría colegir que la desigualdad de oportunidades en la acumulación de capital humano determina los peores resultados en el mercado de trabajo y, en consecuencia, explica mayormente la brecha salarial. El análisis se llevará a cabo para el agregado de las principales trece áreas metropolitanas en Colombia con base en la Encuesta Nacional de Hogares de Diciembre de 2000 (ENH-110), que es la única encuesta especializada en el mercado de trabajo que incluye al unísono un módulo de autorreconocimiento étnico-racial y caracterización social.

\section{Revisión de literatura}

Hay una gran tradición de estudios sobre movilidad intergeneracional, los cuales intentan explicar la desigualdad de oportunidades como resultado de la transmisión intergeneracional del estatus socioeconómico de padres a hijos. Dichos estudios, cuyas contribuciones seminales corresponden a Becker y Tomes (1979) y Loury (1981), examinan la persistencia de las desigualdades por medio de las generaciones por los efectos que tiene el ingreso de los padres sobre la educación de los hijos. Se estima que la escolaridad de los padres es el principal canal en la transmisión intergeneracional del estatus socioeconómico. Padres con mejores niveles educativos pueden tener acceso a recursos financieros para ofrecer calidad y continuidad en las carreras educativas de los hijos (Chevalier, 2004). De hecho, en esos modelos los padres invierten 
directamente en la educación de los hijos, en un nivel que determina el ingreso de la próxima generación. Por último, Bowles (1973) ha distinguido tres formas de la transmisión intergeneracional de estatus socioeconómico: la primera se refiere a las desigualdades en las oportunidades de educación; la segunda explicación enfatiza en que las aspiraciones, características de personalidad y valores de los individuos son fuertemente afectados por las características de la familia y modelos culturales dominantes en la sociedad; por último, se destaca en la transmisión intergeneracional las habilidades intelectuales de padres a hijos.

Por otra parte, el análisis de la transmisión intergeneracional del estatus socioeconómico familiar se ha visto favorecido por la gran producción de estudios sobre discriminación racial principalmente en los Estados Unidos. A este respecto, en América Latina, específicamente en Brasil, ha habido de manera reciente una buena producción de estudios que tratan de indagar el efecto de la discriminación racial sobre la transmisión intergeneracional de las desigualdades, motivados por las grandes diferencias en el ingreso, desigualdad económica y las brechas sociales para la población negra en ese país.

En relación con esto, Soares (2000) estudia la evolución y efecto de la discriminación por raza y sexo en los diferenciales de ingresos en Brasil. Con base en la metodología de descomposición de Oaxaca-Blinder, mediante los diferentes puntos de la distribución del ingreso, encuentran que existe una parte de los diferenciales de ingreso en Brasil que podría atribuirse a la discriminación por raza y sexo.

Campante et al. (2004) incorporan nuevas variables de control que permiten evaluar mejor el efecto de la discriminación racial en los diferenciales de ingresos en ese país. Para ello, incluyen el nivel educativo de los padres debido a la fuerte correlación entre la educación de los padres y los resultados obtenidos por los hijos como la literatura internacional sugiere. Se encontró que al incluir el nivel educativo de la madre en la estimación se reduce el componente de discriminación en las diferencias de ingreso. Adicionalmente, se identificó y cuantificó la discriminación racial entre blancos y no blancos utilizando métodos de descomposición para observar la parte de las diferencias que son atribuidas a las circunstancias, de las que están asociadas con retornos diferenciales de los esfuerzos.

Leite (2005) analiza el impacto del estatus socioeconómico familiar y la calidad de educación que recibieron los individuos en los diferenciales de ingresos. Para ello, utiliza variables instrumentales como la educación de los padres y los test de proficiencia escolar en las municipalidades de Brasil. Los resultados sugieren que en Brasil la brecha de ingresos entre individuos negros y blancos se explica en mayor medida por las restricciones en el acceso 
a recursos de los padres que pudieron incidir en las elecciones educativas de los hijos, lo cual determina una posición ocupacional más precaria y a partir de ello salarios inferiores. No obstante, se comprobó también que una parte de la brecha salarial no se explica por la desigualdad de oportunidades sino por la presencia de mecanismos institucionales en el mercado de trabajo que pueden ser interpretados como discriminación por raza.

Por su parte, en Colombia la mayoría de estudios sobre movilidad intergeneracional han estado usualmente referidos a la transmisión intergeneracional de la educación entre padres e hijos, y a los efectos de la educación de los padres en el logro educativo de los hijos. En este sentido, uno de los primeros trabajos fue el de Nina y Grillo (2000), quienes efectuaron una investigación sobre la movilidad intergeneracional educativa, ocupacional y de ingresos en Colombia. Después, Behrman, Gaviria y Székely (2001) realizaron un análisis de la movilidad intergeneracional educativa y ocupacional en Colombia, Brasil, México, Perú y para Estados Unidos; estudio novedoso por su carácter comparativo entre países. Gaviria (2002) hizo una ampliación al estudio de Behrman, Gaviria y Székely (2001) e introdujo otros factores asociados con las características circunstanciales que pueden afectar la movilidad educativa intergeneracional y ocupacional en Colombia. Cartagena (2004) efectuó un estudio sobre la movilidad educativa entre generaciones en Colombia entre 1915 y 2003. Tenjo (2004) intentó observar cuál es el efecto de la educación de los padres en el logro educativo de los hijos. Viáfara $(2005,2006)$ y Viáfara y Urrea (2006) auscultan el efecto de la raza y de los orígenes sociales en las oportunidades educativas en Colombia. Bonilla (2010) introduce el análisis de la condición migratoria y la zona de residencia en la movilidad educativa intergeneracional. Por último, Viáfara et al. (2010) investigan el efecto de la condición étnico-racial en la movilidad educativa intergeneracional en Colombia. Según Bourguignon et al. (2003, p. 3), pese a la importancia de esta aproximación para medir las desigualdades en las oportunidades de educación entre grupos sociales "[...] esos estudios se enfocan en la movilidad futura esperada, en otras palabras, entre las ganancias potenciales de niños cuando ellos son adultos y las ganancias potenciales de sus padres" (las cursivas son del autor de este artículo).

Desde otra perspectiva, el trabajo de Núñez et al. (2006) es el primero que indaga sobre el efecto del estatus socioeconómico familiar en las ganancias de los individuos. Utilizando la metodología de Bourguignon et al. (2003), los autores intentan analizar la relación que prevalece entre desigualdad de oportunidades y desigualdad de resultados entre hombres y mujeres y determinar en qué medida los niveles observados, en indicadores como los coeficientes de Gini y Theil, pueden atribuirse a variables relacionadas con características 
propias de los individuos y de sus familias. Para esta investigación, utilizan la Encuesta de Calidad de Vida 2003. Los resultados indican que si la educación y los ingresos dependen de características heredadas, la desigualdad se transmite de generación en generación. Cuando se realiza una simulación en donde los individuos presentan las mismas oportunidades, debido a las mismas condiciones iniciales o heredadas, la distribución del ingreso mejora notablemente, conduciendo a una disminución en la brecha salarial.

Ferreira y Meléndez (2010) emplean las encuestas de calidad de vida 1997, 2003, 2008 y 2010 para analizar la desigualdad de resultados y oportunidades en Colombia. Calculan índices de desigualdad de oportunidades para el gasto per cápita del hogar, ingreso del hogar e ingreso laboral, con base en la diferencia entre el índice de desigualdad de Theil (0) calculado sobre la variable resultado y el índice de desigualdad de Theil (0) calculado sobre la distribución (simulada) que resultaría si todos los individuos de la sociedad tuviesen idénticas circunstancias. Los autores demuestran que aproximadamente el 23,6\% de la desigualdad en el gasto per cápita en Colombia es explicada por las circunstancias que tienen las personas al nacer. Con respecto al ingreso per cápita, cerca de 17,6\% de la desigualdad tiene origen en la desigualdad de oportunidades. Sin embargo, los autores argumentan que estos resultados hay que observarlos como un límite inferior en el sentido que no fue posible incluir todas las variables de circunstancias por lo que la desigualdad de oportunidades puede incluso ser mayor, aunque resaltan que esta disminuyó entre 1997 y 2010.

Ruiz (2011) intenta descomponer la contribución de los esfuerzos y las circunstancias sobre la desigualdad de los ingresos en Colombia. Con base en la Encuesta de Calidad de Vida 2008, emplea la metodología propuesta por Bourguignon et al. (2007) y por Ferreira y Gignoux (2008). Los resultados indican que tanto la educación de la madre como del padre son las circunstancias que más explican la desigualdad del ingreso: cerca de $22 \%$ es explicado por estos dos factores, mientras que la zona de nacimiento es otra circunstancia importante que puede explicar alrededor de $7 \%$ a $11 \%$ de la desigualdad de los ingresos.

Galvis-Aponte y Meisel-roca (2014) analizan la movilidad social en Colombia desde una perspectiva regional, para ello utilizan la metodología propuesta por Ferreria y Gignoux (2008), así como índices de condiciones materiales e índices de movilidad intergeneracional en educación. Los resultados muestran que las regiones de Colombia tienen bajos niveles de movilidad intergeneracional en educación. No obstante, los autores encontraron que cuando se comparan los resultados de los años 2003 y 2010 se constatan 
mejoras. Así mismo, se encontró evidencia sobre la existencia de transmisión intergeneracional del logro en condiciones materiales.

\section{Metodología ${ }^{2}$}

El cálculo de la discriminación en el sentido "típico" —-método residual— se realiza teniendo en cuenta los datos sobre los años de escolaridad, experiencia en el trabajo, ocupación o rama de actividad, residencia geográfica, etc., los cuales se usan para establecer los salarios esperados en ausencia de discriminación; la brecha de ganancias "no explicada" es entonces atribuida a la discriminación. Este artículo va mucho más adelante con base en la metodología utilizada por Bourguignon et al. (2003) y Leite (2005).

Como se ha manifestado con anterioridad, las diferencias de ingreso en el mercado laboral se pueden explicar con base en las características personales como la raza, el sexo y estatus socioeconómico familiar, las cuáles se asocian con las circunstancias. Además, por el efecto de otras variables que tienen que ver con el capital humano acumulado - una vez se ha tomado decisiones propias en su educación formal-, capacitación en el trabajo, la experiencia, las decisiones anteriores de cambios de trabajo o de región de residencia, que están relacionadas con los esfuerzos. No obstante, como lo comenta Bourguignon et al. (2003, p. 2), uno de los factores que han restringido el trabajo empírico sobre la desigualdad de oportunidades, además de la limitada disponibilidad de variables que permitan medir las circunstancias, es la dificultad conceptual de separar esfuerzos y circunstancias. "Tal conocimiento ayudaría a definir el alcance actual de las políticas de redistribución y en particular debería de informar en torno a la elección entre redistribuir el ingreso corriente o expandir un conjunto de oportunidades para los pobres; por ejemplo, a través de la confección de una estrategia de acumulación de capital humano entre niños, menos dependientes de sus padres".

\section{1. ¿Cómo separar el efecto de las circunstancias y esfuerzos en la brecha salarial?}

El cálculo de la discriminación en el sentido "típico" tiene el inconveniente que no permite separar el efecto de la transmisión intergeneracional del capital humano del efecto de la discriminación. Esto sucede, básicamente, por el sesgo al estimar el modelo sin instrumentos para la escolaridad de los

2 Esta sección sigue muy cerca de Leite (2005). 
individuos, asociado con la correlación entre la escolaridad y con el estatus socioeconómico familiar en el modelo "típico" utilizando Mínimos Cuadrados Ordinarios (MCO) (Leite, 2005).

Para corregir este sesgo, debido a la omisión de variables no observadas (escolaridad de los padres y otras) que están correlacionadas con el nivel de educación de los individuos, se puede utilizar una o varias variables instrumentales. Esto se puede hacer recurriendo al método de Mínimos Cuadrados Ordinarios en dos Etapas (MC2E) en lugar de Mínimos Cuadrados Ordinarios.

A continuación se describe el modelo:

$$
\begin{aligned}
\ln w & =\beta . E d+X \cdot y_{1}+\varepsilon \\
E_{d} & =Z \cdot \pi+X \cdot \lambda+v
\end{aligned}
$$

Donde $\mathrm{w}$ es el salario del individuo, $E d$ es el nivel educativo, $\mathrm{Z}$ es un vector que contiene la educación de los padres (instrumento) y $X$ es un control de vectores con otra serie de características individuales como el color de la piel, género, edad, estructura del hogar, $\varepsilon$ y $v$ son los vectores de error no observados.

Asumiendo que $Z$ está correlacionado con $E d$ y no con $v$, es posible obtener una inferencia no sesgada en $\beta$ y $y_{1}$ El supuesto de identificación es que las variables exógenas no aparecen en la ecuación de salarios. El modelo reducido se puede reescribir de la siguiente manera:

$$
\mathrm{Y}=\mathrm{Z} \cdot \pi \cdot a+\mathrm{X} \eta+V
$$

Donde

$$
\begin{gathered}
Y=[\ln w, E d], Z=[E d P a r], V=\left[v_{t}, v\right], a=[\beta ; 1], \eta=[\gamma, \lambda] \\
\mathrm{v}_{\mathrm{t}}=\varepsilon+v \beta ; \gamma=\gamma_{1}+\lambda \beta
\end{gathered}
$$

A pesar de la relevancia del modelo MC2E de variables instrumentales, se decide no solamente buscar el coeficiente de discriminación que se obtiene de los modelos "típicos" a partir de la utilización de una variable dummy sobre el color de la piel (por ejemplo, blanco =1, negro =0). En lugar de esto, se plantea la realización de microsimulaciones para observar los logros potenciales de los negros bajo la hipótesis de que ellos son pagados como los blancos. Así, la metodología empleada es una adaptación del procedimiento Oaxaca 
- Blinder (Oaxaca, 1973 y Blinder,1973). Considerando a W como blancos y B como negros, se modelará separadamente blancos y negros con el objetivo de estimar los precios para ambos grupos de población y luego utilizar los parámetros estimados de los blancos sobre las características de los negros para estimar cómo sería el salario de los negros si estos fueran pagados como blancos. Matemáticamente, la simulación se define como:

Modelo 1

$$
Y_{B}^{*}=Z_{B} \cdot \hat{\pi}_{B} \cdot \hat{a}_{w}{ }^{\prime}+X_{B} \hat{\eta}+V
$$

Donde

$$
\begin{gathered}
Y_{B}^{*}=\left[\ln W_{B}^{*} ; Z_{B}\right] Z_{B}=\left[\operatorname{EdPar}_{B} ; P T_{B} ; S T_{B}\right], V=\left[\varepsilon_{B}+v_{B} \hat{\beta}_{w} ; v\right] \\
\hat{a}_{w}{ }^{\prime}=\left[\hat{\beta}_{w} ; 1\right]^{\prime}, \hat{\eta}=\left[\hat{\lambda}_{1 w}+\hat{\lambda}_{B} \hat{\beta}_{w} ; \hat{\lambda}_{B}\right]
\end{gathered}
$$

Modelo 2

$$
Y_{B}^{*}=Z_{B} \cdot \hat{\pi}_{w} \cdot \hat{a}_{w}{ }^{\prime}+X_{B} \hat{\eta}+V
$$

Donde

$$
\begin{gathered}
Y_{B}^{*}=\left[\ln W_{B}^{*} ; Z_{B}\right] Z_{B}=\left[\operatorname{EdPar}_{B} ; P T_{B} ; S T_{B}\right], V=\varepsilon_{B}+v_{B} \hat{\beta}_{w} ; v \\
\hat{a}_{w}{ }^{\prime}=\left[\hat{\beta}_{w} ; 1\right], \hat{\eta}=\left[\hat{\lambda}_{1 w}+\hat{\lambda}_{w} \hat{\beta}_{w} ; \hat{\lambda}_{w}\right]
\end{gathered}
$$

Además, después de estimar la ecuación (3), cambiando solamente un conjunto de parámetros en la ecuación de salarios, es fácil obtener la brecha salarial con la siguiente descomposición:

$$
\Delta=\bar{Y}_{w}-\bar{Y}_{B}=\left(\bar{Y}_{w}-\bar{Y}_{B}^{*}\right)+\left(\bar{Y}_{B}^{*}-\bar{Y}_{B}\right)-\Delta_{1}+\Delta_{2}
$$

El primer término del lado derecho corresponde a la parte del diferencial de salarios que se atribuye a las circunstancias, puesto que ello presenta la 
comparación entre los salarios estimados de los dos grupos de individuos bajo los mismos parámetros, el cual se llama efecto de las circunstancias. El segundo término, por otro lado, indica cuánto del salario de los individuos negros varía debido a esos parámetros. Esto se debe al efecto precio, que indica que tan diferentemente son premiados los esfuerzos de los individuos blancos, relativo a los individuos negros. En el caso de la ecuación (4), la descomposición no es simple porque se mezclan los efectos de las circunstancias y precios. Lo anterior se denomina el efecto global.

Además de la descomposición, la técnica propuesta permite obtener la distribución de densidad de los salarios porque únicamente no trata con el promedio de características como la aproximación de Oaxaca-Blinder sino también con la distribución total de los salarios.

Es importante advertir que según Dadaby et al. (2004), este tipo de análisis tiene limitaciones asociadas con la lógica y supuestos subyacentes a la inferencia causal entre raza y discriminación. A este respecto, Fortín et al. (2011) han señalado algunos problemas fundamentales: (i) es una descomposición secuencial, lo que implica que el orden afectará las conclusiones; (ii) la técnica solo es útil para las descomposiciones de las diferencias promedio; (iii) esta técnica es intrínsecamente un enfoque de equilibrio parcial. Esto último significa que aumentar el capital humano de las personas negras al nivel de las blancas no tiene ningún efecto en los retornos, es decir, los parámetros son independientes de las cantidades. En este caso el aumento del capital humano pudiera deprimir los retornos e invalidar la descomposición.

No obstante, debido a que es difícil observar el comportamiento discriminatorio de manera directa, los investigadores deben recurrir a varias alternativas metodológicas para su estudio. En este caso, se debe suponer que los peores resultados para un individuo habrían sido diferentes si el individuo hubiera sido de un color de piel diferente.

\subsection{Datos}

La información utilizada en este artículo se refiere a la Población Económicamente Activa (PEA) de la Encuesta Nacional de Hogares del Dane para el cuarto trimestre del año 2000, ENH etapa 110, para las trece principales áreas metropolitanas de Colombia. La razón fundamental para utilizar esta encuesta se debe a la presencia del módulo de caracterización social que contiene información sobre el ambiente socioeconómico familiar, así como por la técnica de autoclasificación en términos étnico-raciales a partir de la utilización de 4 fotografías que dan una aproximación al color de la piel de las personas. Es importante aludir que esta es la única encuesta de hogares en Colombia 
que permite hacer el ejercicio propuesto. El Dane solo incluyó un módulo de autorreconocimiento étnico-racial en la ECH del segundo trimestre de 2004 y en la GEIH de 2006-2007, pero la validez externa de esas encuestas para los grupos étnicos-raciales es muy cuestionable, además de que no incluyen variables asociadas con las circunstancias en las cuales un individuo nace.

La muestra está compuesta por 56240 personas económicamente activas. De esta muestra, se seleccionó una submuestra de 40560 personas entre 16 y 68 años de edad, ocupadas con veinte o más horas trabajadas a la semana.

En el estudio se incluye una variable de esfuerzo asociada con la persona, como son los años de educación alcanzados y la edad, una variable demográfica (como proxy a la experiencia potencial). Además, algunas asociadas con las circunstancias como el nivel educativo de los padres, presencia del padre en el hogar, jefatura del hogar, género y el color de la piel de las personas. Estas variables se definen de la siguiente manera:

Educación: mide el número de años de educación aprobados. Corresponde a los años de escolaridad alcanzados por la persona. Con esta variable se intenta dar cuenta de las capacidades, conocimientos, competencias y calificaciones de las personas.

Edad: número de años con que cuenta la persona en el momento de la encuesta. Es una variable proxy de la experiencia potencial de la persona en el mercado de trabajo.

Jefatura de hogar: variable dicotómica que toma el valor de uno para los jefes del hogar y cero en otro caso.

Nivel educativo de los padres: es el máximo nivel educativo alcanzado por el padre. Se codifica como 0 para las personas con padres con ningún nivel educativo, 1 "primaria", 2 "secundaria" y 3 "universitaria". Es una variable proxy del ambiente socioeconómico familiar de las personas. Lo anterior significa que la educación de los padres va a estar asociada con las oportunidades de logro educativo de las personas y desde aquí van a determinar los diferentes esfuerzos que tienen que hacer los individuos para alcanzar un determinado nivel educativo.

Presencia del padre en el hogar: variable dicotómica que toma el valor de uno si el padre vive en el hogar y cero en otro caso.

Género: variable dicotómica que toma el valor de 0 para las mujeres y 1 para los hombres.

Color de la piel: corresponde a la autoidentificación de las personas de sus rasgos fenotípicos sobre la base de cinco fotografías como se muestra en el Anexo 1. Se codifica como 0 para las personas que se autoidentificaron como negras, 1 para los mulatos, 2 para los mestizos y 3 para los blancos. 


\section{Resultados}

\subsection{Hechos estilizados}

Para empezar, la distribución de los niveles de educación de los padres según color de la piel es muy desigual en Colombia. Como puede observarse, la población negra tiene una mayor proporción de padres con niveles de educación inferiores; para los otros grupos étnico-raciales la participación en estos niveles de educación se reduce de manera monótona a medida que el color de la piel se hace más claro. De manera inversa, la población negra tiene la participación más baja en los niveles de educación superiores, que se incrementan para la población con colores de piel más clara (figura1). Esto significa que la población negra ostenta un menor estatus socioeconómico familiar que se relaciona con sus orígenes sociales más empobrecidos; los orígenes sociales se mejoran en la medida que el color de la piel se hace más claro. Como resultado de la fuerte asociación entre el estatus socioeconómico familiar y las oportunidades educativas, la población negra enfrentaría más limitaciones financieras para el disfrute de carreras educativas largas y de calidad, y no menos importante, en la transferencia de habilidades cognitivas entre padres e hijos (Bowles, 1973).

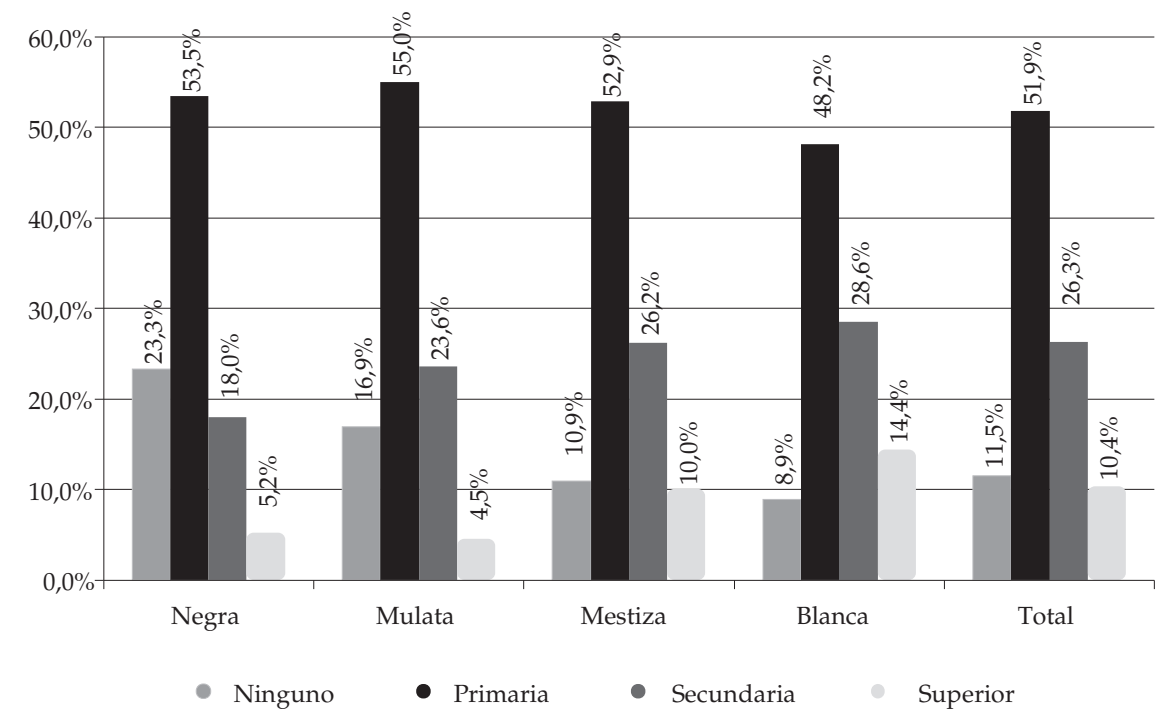

Figura 1. Nivel educativo del padre según color de la piel

Fuente: elaboración propia con base en la Encuesta Nacional de Hogares 2000 -etapa 110 
Por otra parte, y en consonancia con el menor estatus socioeconómico familiar, los años de educación tienen una relación inversa con el color de la piel de las personas en Colombia. Es decir, mientras que para del agregado de la población colombiana en las principales áreas metropolitanas el promedio de años de educación alcanzó 8,65 años, los menores registros se presentaron para la población negra con apenas 6,76, y en el otro extremo la población blanca con 9,28; la población mulata y mestiza se ubicaron simétricamente en la mitad con 8,72 años y 7,52 años de escolaridad, respectivamente. Lo anterior equivale a una brecha en años de educación de la población negra con respecto a la población mulata, mestiza y blanca de $11,24 \%, 28,9 \%$ y $37,2 \%$, respectivamente (figura 2).

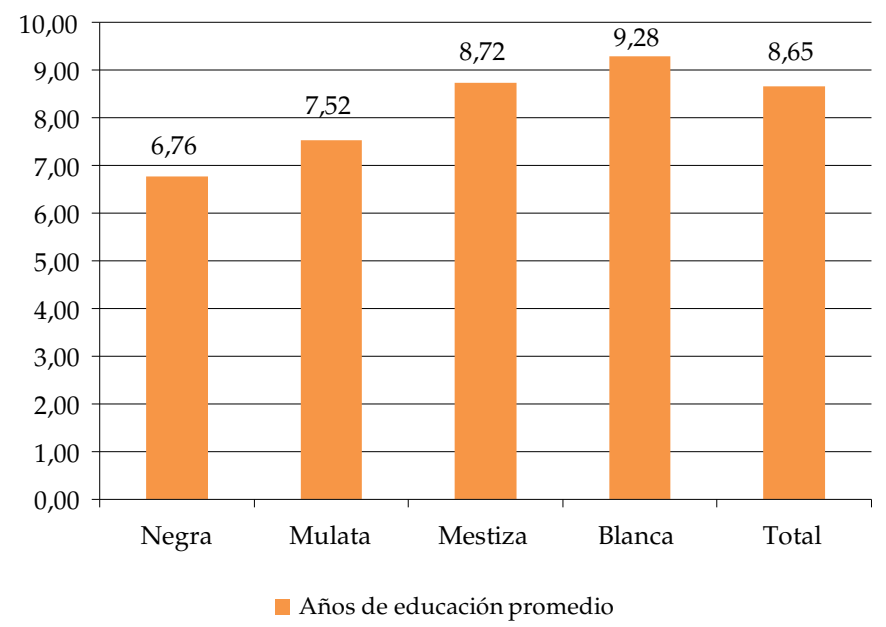

Figura 2. Años promedio de educación según el color de la piel Fuente: elaboración propia con base en la Encuesta Nacional de Hogares 2000 -etapa 110.

De otro lado, en consonancia con los menores años de educación, la población negra ostenta menores ingresos promedio por hora; los ingresos muestran una relación inversa con el color de la piel de las personas en Colombia. En concreto, el ingreso promedio por hora de la población negra es alrededor de $73,40 \%$ más bajo que el de la población blanca y $57,73 \%$ y $8,45 \%$ cuando es comparada con la población mestiza y mulata, respectivamente (figura 2).

En este mismo orden y dirección, la participación de la población colombiana en los diferentes quintiles de ingreso exhibe diferencias importantes de acuerdo con el color de la piel. La población negra exhibe una participación más alta en los quintiles de ingresos más bajos y en sentido inverso la menor participación en el techo de la distribución del ingreso. Esas participaciones 
se reducen, para el quintil inferior, y se incrementan para el quintil superior, a medida de que el color de la piel se hace más claro (figura 4).

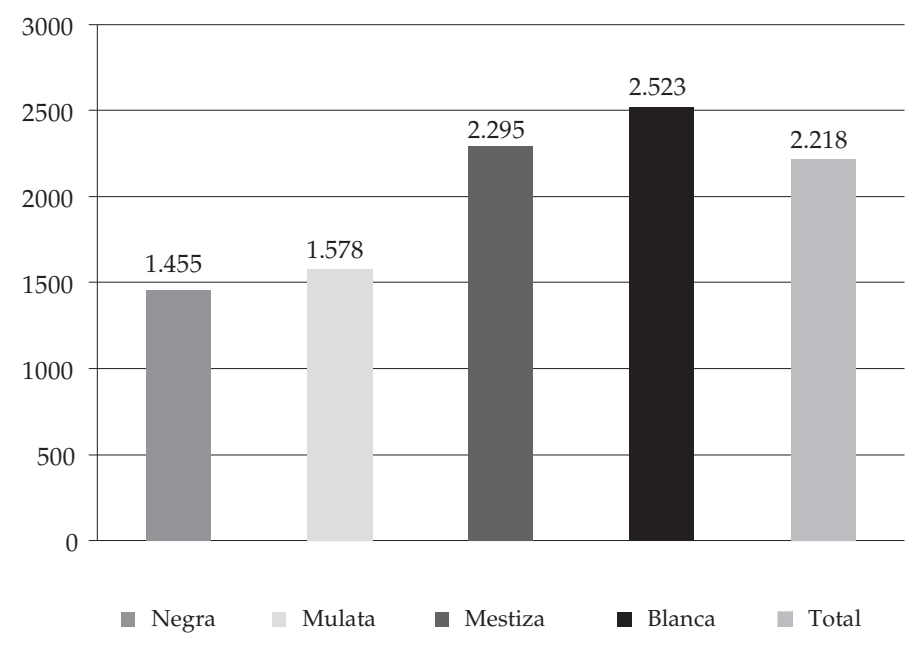

Figura 3. Ingreso laboral por hora según el color de la piel

Fuente: elaboración propia con base en la Encuesta Nacional de Hogares 2000 -etapa 110.

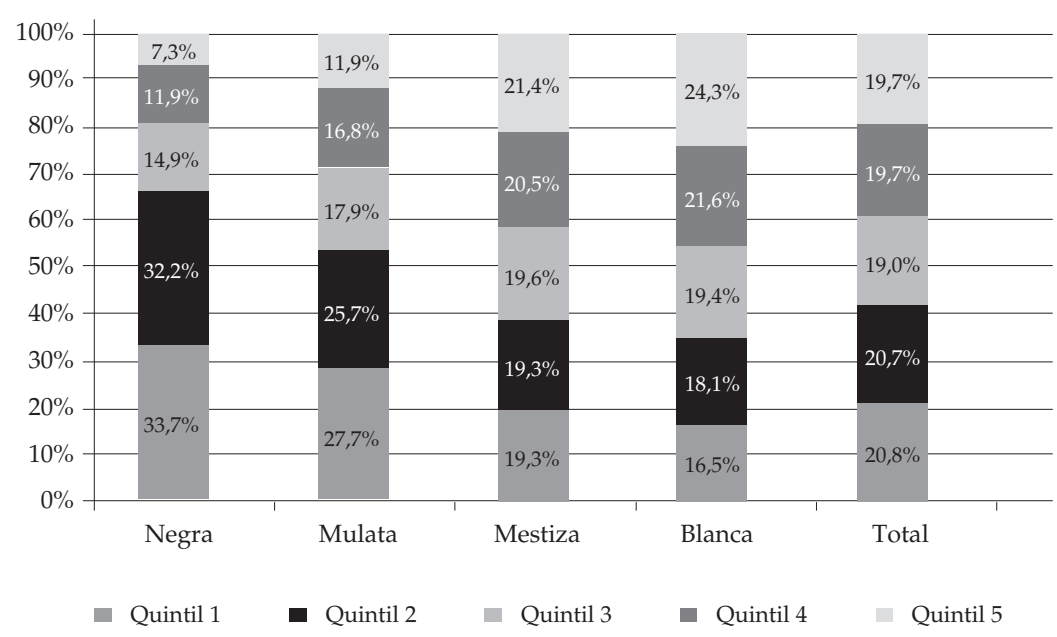

Figura 4. Quintiles de ingreso laboral según el color de la piel Fuente: elaboración propia con base en la Encuesta Nacional de Hogares 2000 -etapa 110.

Por contraste, el número de horas trabajadas de la población en Colombia según grupos étnico-raciales no muestra diferencias significativas. Esto es, la mayoría de la población se ubica cerca del promedio Nacional $(47,92)$, 
aunque es importante señalar que inclusive las horas trabajadas de la población negra y la población mulata son ligeramente más altas, 48,93 y 48,52, respectivamente (figura 5).

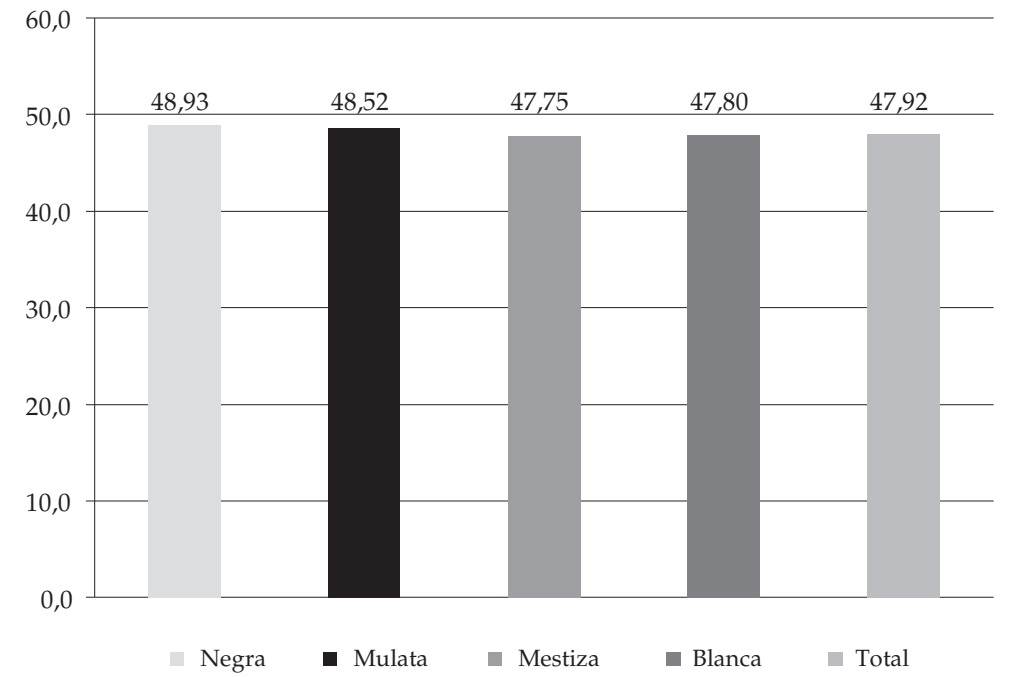

Figura 5. Horas trabajadas en promedio a la semana según color de la piel Fuente: elaboración propia con base en la Encuesta Nacional de Hogares 2000 -etapa 110.

Con todo, se observan diferenciales importantes en las características circunstanciales del mercado de trabajo de la población según el color de la piel en Colombia. No obstante, el análisis univariado no permite sacar conclusiones sobre el efecto de estas características circunstanciales frente a la valoración de los esfuerzos en la determinación de las diferencias de ingreso. En concreto, los resultados sugieren que una persona con color de piel más oscuro que otra podría tener más limitaciones en las elecciones educativas de sus hijos debido a una mayor carencia de recursos. Además, las amplias brechas de ingreso por color de la piel probablemente también se producen por la presencia de mecanismos institucionales en el mercado de trabajo como la discriminación. Esto significa que hay desigualdades importantes en el ambiente familiar que es importante captar en la ecuación de salarios para observar con mayor claridad qué tanto de las brechas de ingreso según el color de la piel en Colombia están asociadas con una valoración desigual e injusta por los esfuerzos, o qué tanto a la desigualdad de oportunidades asociadas con las características circunstanciales.

A los efectos de este, el instrumento principal para controlar la endogeneidad de la educación del individuo es la educación del padre. Una manera 
de valorar la pertinencia del instrumento es observar su correlación con la variable explicativa endógena. La tabla 1 contiene las correlaciones parciales y los coeficientes de Spearman, que fueron todos estadísticamente significativos a un $1 \%$. Obsérvese que si un padre tiene primaria está relacionado de manera negativa con los años de educación que alcanza su hijo, mientras que si un padre tiene secundaria o educación superior se relaciona de manera positiva con los años de educación alcanzados por la persona.

Tabla 1. Correlaciones de Spearman y de Pearson entre los años de educación del individuo y el nivel educativo del padre

\begin{tabular}{lcc}
\hline & Corr. Spearman & Corr. Pearson \\
\hline Primaria & $-0,0942^{* * *}$ & $-0,0866^{* * *}$ \\
Secundaria & $0,2383^{* * *}$ & $0,2331^{* * *}$ \\
Superior & $0,2216^{* * *}$ & $0,2275^{* * *}$ \\
\hline
\end{tabular}

Nota: ${ }^{* * *} \mathrm{p}<0,01,{ }^{* *} \mathrm{p}<0,05,{ }^{*} \mathrm{p}<0,1$.

Por otro lado, la prueba Pearson chi-cuadrado para mirar la relación de asociación entre dos variables categóricas, en este caso los niveles educativos entre el padre y el hijo fue igual a Chi(9) $=1.90 \mathrm{E}+04$ con P-valor de 0,000 , lo que indica que bajo la hipótesis de no asociación entre los niveles educativos no se puede rechazar la hipótesis nula; en otras palabras, existe relación entre la educación de los hijos y de los padres.

Desde otra perspectiva, Andrews et al. (2004) argumentan que se debe practicar una prueba de debilidad cuando el instrumento está débilmente correlacionado con la variable explicativa endógena. En el Anexo 2 se muestran diferentes pruebas de debilidad del instrumento para el logro educativo del individuo, y se encuentra que los instrumentos empleados son importantes para explicar la educación del individuo. Los instrumentos empleados son los niveles educativos del padre, el área metropolitana en la que vive el individuo, el género, si la persona es jefe del hogar y si el padre del individuo vive en el hogar.

\subsection{Estimación de modelos econométricos}

A continuación, se presentan las estimaciones de la ecuación 2. Los modelos presentados se conocen en la literatura como modelos raza-intercepto (Blank, Dabady \& Citro, 2004). La variable dependiente es el logaritmo natural del ingreso hora. Como variables independientes se incluyeron la educación, la edad, la edad al cuadrado, la jefatura del hogar, el género y el color de la 
piel. Además, con el objetivo de corregir los posibles problemas de sesgo de selección presentes en las ecuaciones mincerianas, se incluyó la razón inversa de Mills propuesta por Heckman (1998). ${ }^{3}$

En este propósito, en las tablas a continuación en el panel A se presenta la estimación por MCO, y en el panel B la estimación por MC2E. En cada uno de los paneles la primera columna corresponde a los coeficientes, después se presenta el error estándar y luego los p-valores asociados con cada una de las variables. En cada uno de los modelos estimados, las personas negras constituyen la población de referencia en comparación con la población mulata (tabla 2), la población mestiza (tabla 3) y la población blanca (tabla 4). Se advierte que la exposición de los resultados se centra en el efecto del color de la piel en los diferenciales de ingreso que es el objetivo fundamental de este artículo.

Para empezar, la tabla 2 presenta las estimaciones de los modelos para la población mulata y negra. En primer término, el coeficiente que mide la brecha salarial entre las personas mulatas y negras muestra que ser mulato frente a ser negro incrementa el salario en $5,07 \%$. Con respecto al coeficiente de la educación indica que un año adicional de educación aumentaría el ingreso laboral por hora en $8,9 \%$. Por su parte, en el modelo MC2E se observa claramente que existe una caída aproximada del $0,8 \%$ en el coeficiente asociado con el color de la piel, tal como se esperaba (pasa de 5,07\% a 4,23\%), mientras los retornos a la educación aumentaron en cerca de 1,4\%.

Tabla 2. Estimaciones de las ecuaciones de ingreso entre la población Mulata y Negra por MCO y MC2E

\begin{tabular}{lccccccc}
\hline \multirow{2}{*}{$\begin{array}{c}\text { Variable dependiente: } \\
\text { logaritmo del salario por hora }\end{array}$} & Coeficiente & Std & P-value & Coeficiente & Std & P-value \\
\cline { 2 - 7 } & 0,0892 & 0,0019 & 0 & 0,1028 & 0,0068 & 0 \\
\hline Años de educación & 0,0483 & 0,0037 & 0 & 0,0375 & 0,0052 & 0 \\
Experiencia & $-0,0004$ & 0,0000 & 0 & $-0,0003$ & 0,0001 & 0 \\
Experiencia 2 & 0,0591 & 0,0267 & 0,027 & 0,1244 & 0,0408 & 0,002 \\
Jefe del hogar & 0,0778 & 0,0269 & 0,004 & 0,0344 & 0,0381 & 0,366 \\
Sexo == hombre & & & & & & Continúa
\end{tabular}

3 Con esta especificación se pretende estimar un modelo más cercano a la ecuación original de Mincer. Además, no se incluyeron variables de control como la ocupación u otras características del empleo porque podrían estar correlacionadas con la discriminación. Variables regionales son importantes pero la muestra solo es representativa para grupos étnico-raciales en el ámbito general. 


\begin{tabular}{lccccccc}
\hline & \multicolumn{5}{c}{ MCO } & \multicolumn{4}{c}{ MC2E } \\
\cline { 2 - 7 } $\begin{array}{l}\text { Variable dependiente: } \\
\text { logaritmo del salario por hora }\end{array}$ & Coeficiente & Std & P-value & Coeficiente & Std & P-value \\
\hline Mulata - Negra & 0,0507 & 0,0184 & 0,006 & 0,0423 & 0,0299 & 0,157 \\
Razón Inversa de Mills & $-0,1045$ & 0,0357 & 0,003 & $-0,0527$ & 0,0547 & 0,335 \\
Constante & 1,0224 & 0,1011 & 0 & 1,0840 & 0,1467 & 0 \\
R-Cuadrado & & 0,3081 & & & 0,2926 & \\
\# Observaciones & & 8468 & & & 6801 & \\
\hline
\end{tabular}

Fuente: elaboración propia con base en la Encuesta Nacional de Hogares 2000 -etapa 110.

Para continuar, la tabla 3 presenta las estimaciones de los modelos para la población mestiza y negra. Por MCO, el coeficiente del color de la piel es de 12,67\%; igualmente, la tasa de retorno de la educación es de alrededor 9,85\%. Por MC2E, el efecto del color de la piel se reduce a $8,17 \%$, y, al contrario, la tasa de retorno de la educación se incrementa a cerca de $15 \%$.

Seguido a esto, la tabla 4 presenta las estimaciones de los modelos para la población blanca y negra. Obsérvese que por MCO el coeficiente asociado con el color de la piel es de 14,3\%; en igual forma, el efecto de un año adicional de educación es de 9,53\%. Por MC2E, el coeficiente del color de la piel se reduce a $7,45 \%$; la tasa de retorno a la educación se incrementa a $14.6 \%$.

Los resultados anteriores permiten observar un patrón de comportamiento de las brechas de ingreso según el color de la piel de las personas. Como puede observarse, los diferenciales de ingreso de la población negra frente a los otros grupos étnico-raciales se incrementan a medida de que el color de la piel se hace más claro. De hecho, la brecha de ingresos más pequeña de la población negra es frente a la población mulata $(5,07 \%)$, mientras la brecha más grande es frente a la población blanca con (14,3\%); la población mestiza se ubica simétricamente en la mitad con una brecha de $(12,67 \%)$.

Por otra parte, cuando se corrige el sesgo de endogeneidad, las brechas de ingreso según el color de la piel se reducen de manera importante. Lo anterior se produce debido a que ahora se está contabilizando la transmisión intergeneracional del capital humano. Como las personas negras tienen menor background, esto incrementa sus retornos frente a aquellas personas que tienen iguales años de educación pero con mejor background, lo cual reduce el posible efecto de la discriminación de mercado en las brechas de ingreso. Más aún, los diferenciales entre ambos coeficientes se incrementan cuanto más grandes sean los diferenciales en el background, como se puede constatar. Estos resultados van en la misma dirección de las variaciones presentadas en 
los modelos estimados por Leite (2005) y son acordes a la evidencia empírica suministrada por Card (1999).

Tabla 3. Estimaciones de las ecuaciones de ingreso entre la población Mestiza y Negra por MCO y MC2E

\begin{tabular}{lcccccc}
\hline \multirow{2}{*}{$\begin{array}{l}\text { Variable dependiente: Logarit- } \\
\text { mo del salario por hora }\end{array}$} & \multicolumn{3}{c}{ MCO } & \multicolumn{3}{c}{ MC2E } \\
\cline { 2 - 7 } Años de educación & Coeficiente & Std & P-value & Coeficiente & Std & P-value \\
\hline Experiencia & 0,0985 & 0,0018 & 0 & 0,1458 & 0,0084 & 0 \\
Experiencia 2 & 0,0507 & 0,0026 & 0 & 0,0524 & 0,0041 & 0 \\
Jefe del hogar & $-0,0004$ & 0,0000 & 0 & $-0,0005$ & 0,0001 & 0 \\
Sexo =- Hombre & 0,0357 & 0,0241 & 0,138 & 0,5356 & 0,0813 & 0 \\
Mestiza- Negra & 0,0300 & 0,0167 & 0,073 & 0,3097 & 0,0472 & 0 \\
Razón Inversa de Mills & 0,1267 & 0,0181 & 0 & 0,0817 & 0,0302 & 0,007 \\
Constante & $-0,1715$ & 0,0375 & 0 & 0,7250 & 0,1402 & 0 \\
R-Cuadrado & 0,9758 & 0,0873 & 0 & $-0,6521$ & 0,2890 & 0,024 \\
\# Observaciones & & 0,3497 & & & 0,3342 & \\
\hline
\end{tabular}

Fuente: elaboración propia con base en la Encuesta Nacional de Hogares 2000 -etapa 110.

Tabla 4. Estimaciones de las ecuaciones de ingreso entre la población Blanca y Negra por MCO y MC2E

\begin{tabular}{llrllll}
\hline \multirow{2}{*}{$\begin{array}{l}\text { Variable dependiente: Logarit- } \\
\text { mo del salario por hora }\end{array}$} & \multicolumn{5}{c}{ MCO } & \multicolumn{3}{c}{ MC2E } \\
\cline { 2 - 7 } Coeficiente & Std & P-value & Coeficiente & Std & P-value \\
\hline Años de educación & 0,0953 & 0,0029 & 0 & 0,1463 & 0,0124 & 0 \\
Experiencia & 0,0492 & 0,0035 & 0 & 0,0493 & 0,0057 & 0 \\
Experiencia 2 & $-0,0004$ & 0,0000 & 0 & $-0,0004$ & 0,0001 & 0 \\
Jefe del hogar & 0,0075 & 0,0316 & 0,813 & 0,4655 & 0,1077 & 0 \\
Sexo == Hombre & $-0,0374$ & 0,0228 & 0,101 & 0,2385 & 0,0697 & 0,001 \\
Blanca- Negra & 0,1430 & 0,0192 & 0 & 0,0745 & 0,0329 & 0,024 \\
Razón Inversa de Mills & $-0,2086$ & 0,0493 & 0 & 0,6010 & 0,1925 & 0,002 \\
Constante & 1,1680 & 0,1274 & 0 & $-0,5724$ & 0,4508 & 0,204 \\
R-Cuadrado & 0,3858 & & & 0,3804 & & \\
\# Observaciones & 10076 & & & 8784 & & \\
\hline
\end{tabular}

Fuente: elaboración propia con base en la Encuesta Nacional de Hogares 2000 -etapa 110. 


\subsection{La microsimulación}

A continuación, y con el objetivo de separar el efecto de las circunstancias y esfuerzos en los diferenciales de ingreso de personas categorizadas por el color de la piel en Colombia, se presenta la estimación de las ecuaciones 3 y 4, y sobre esta base se realiza la descomposición de la ecuación 5.

Los resultados se presentan en la tabla 5. La primera columna (a) de la parte superior de la tabla presenta el logaritmo del ingreso laboral promedio por hora para el grupo que se compara con la población negra. En la segunda columna (b) se enseña el logaritmo del ingreso laboral promedio por hora para la población negra. La tercera columna (c) muestra el diferencial porcentual observado entre los ingresos de la población mulata, mestiza y blanca, respectivamente, en comparación con la población negra. La cuarta columna (d) exhibe el logaritmo del ingreso promedio por hora que debería obtener la población negra si en el mercado laboral le remunera como a su grupo de referencia. La quinta columna (e) muestra el primer término del lado derecho de la ecuación 5 que no es más que el efecto de las circunstancias, y la siguiente columna (f) es el efecto de los esfuerzos. La última columna (g) representa la participación del efecto precio en el diferencial de ingreso laboral promedio hora entre la población de referencia y la negra.

Para empezar, se realiza la comparación entre las personas mulatas y las personas negras. Como puede observarse, el diferencial de ingreso laboral a favor de las personas mulatas es de $13,10 \%$. Luego, si las personas negras son remuneradas como las personas mulatas su logaritmo de ingreso promedio hora sería 2,82. Como resultado, el efecto de las circunstancias y esfuerzos son $6,50 \%$ y $6,60 \%$, respectivamente. La participación del efecto precio en el diferencial salarial total es 50,38\%. Es decir, los diferenciales de ingreso entre las personas mulatas y las personas negras se producen en combinación entre el efecto de las circunstancias y el efecto de los resultados, pero con una leve mayor importancia del primero.

En segundo lugar, la comparación entre las personas mestizas y las personas negras arroja un diferencial de ingreso laboral promedio hora a favor de las personas mestizas de 39,30\%. En efecto, si a las personas negras se le remunerara como a las personas mestizas su logaritmo de ingreso hora sería 2,89. Mientras tanto, el efecto de las circunstancias y resultados es de 25,3\% y $14,0 \%$, respectivamente. Esto significa que la participación del efecto precio es de $35,6 \%$ sobre el diferencial total y la participación del efecto característica corresponde alrededor del $65 \%$ de los diferenciales en el ingreso laboral promedio hora. 
En tercer lugar, al realizar la comparación entre las personas blancas y las personas negras se observa un diferencial del ingreso laboral promedio hora de $47,10 \%$ a favor de las personas blancas. Cuando a las personas negras se le remunera como a las personas blancas su logaritmo de ingreso hora es 2,88. En consecuencia, el efecto de las circunstancias y resultados es de $34,1 \%$ y $13,0 \%$, respectivamente. Con esto, el efecto precio solo explica el $27,6 \%$ de los diferenciales de ingreso.

Los anteriores resultados revelan que a medida de que la población negra es comparada con personas cuyo color de piel es más clara, las brechas en el ingreso laboral promedio por hora son más grandes. Así mismo, el efecto de las circunstancias es mayor a medida de que el color de la piel se hace más clara, mientras que el efecto de los resultados también se incrementa pero no de manera monótona a medida que el color de la piel se hace más claro, como en el caso del efecto de las características.

Desde otra perspectiva, la parte inferior de la tabla 5 contiene las estimaciones de la ecuación 4 . Cabe recordar que en esta ecuación se permutan, para la población negra, los coeficientes asociados con las características circunstanciales como los retornos a la educación de la población de referencia para obtener un efecto global de la desigualdad de oportunidades y discriminación que enfrenta dicha población. En otras palabras, se estima cuál sería el ingreso potencial de la población negra si enfrenta las mismas circunstancias y retornos a los esfuerzos del grupo de referencia. En primer lugar, el logaritmo del ingreso promedio hora es más grande cuando se incluyen las circunstancias que solo cuando se reemplazan los retornos a los esfuerzos (ecuación 3). En consecuencia, el efecto de la desigualdad de oportunidades para la población negra alcanza $12,7 \%$ frente a la población mulata, $28,8 \%$ frente a la población mestiza y $32,9 \%$ frente a la población blanca. Esto significa que la desigualdad de oportunidades es más grande a medida que el color de la piel es más claro. El hecho de que el delta 1 del panel superior sea menor indica la importancia de la desigualdad de oportunidades en la brecha del salario promedio hora entre grupos sociales diferenciados por el color de la piel.

Visto desde otro ángulo, los gráficos 6 a 8 muestran las distribuciones de la población negra, sus contrapartes y la distribución contrafactual estimados mediante la ecuación 3. Los gráficos exhiben que la distribución contrafactual siempre se encuentra desplazada hacia la derecha en referencia a la distribución de la población negra, lo que indica que la población negra estaría en mejor situación relativa a lo que está si es remunerada como sus contrapartes. Además, los últimos tres gráficos (9-11) muestran que el desplazamiento es notable cuando se tiene en cuenta el efecto intergeneracional y el efecto precios mezclados. 
Tabla 5. Diferencias en el logaritmo del ingreso laboral por hora promedio entre la población negra y demás grupos raciales

\begin{tabular}{|c|c|c|c|c|c|c|c|}
\hline & \multicolumn{3}{|c|}{ Observado } & \multicolumn{4}{|c|}{$\begin{array}{l}\text { Población negra pagada como grupo de refe- } \\
\text { rencia YB* - ecuación (3) }\end{array}$} \\
\hline & A & B & $\mathrm{C}$ & $\mathrm{D}$ & E & $\mathrm{F}$ & G \\
\hline & $\begin{array}{l}\text { Ingreso } \\
\text { otro grupo }\end{array}$ & $\begin{array}{c}\text { Ingreso } \\
\text { negra }\end{array}$ & (a)-(b) & $\mathrm{YB}^{*}$ & $\begin{array}{l}\text { Delta } 1 \\
\text { (a)-(d) }\end{array}$ & $\begin{array}{l}\text { Delta } 2 \\
\text { (d)-(b) }\end{array}$ & $\begin{array}{l}\text { Efecto pre- } \\
\text { cio }(\mathrm{f}) /(\mathrm{c})\end{array}$ \\
\hline Mulata & 2,885 & 2,754 & $13,10 \%$ & 2,82 & $6,50 \%$ & $6,60 \%$ & $50,38 \%$ \\
\hline Mestiza & 3,147 & 2,754 & $39,30 \%$ & 2,894 & $25,30 \%$ & $14,00 \%$ & $35,62 \%$ \\
\hline \multirow[t]{4}{*}{ Blanca } & 3,225 & 2,754 & $47,10 \%$ & 2,884 & $34,10 \%$ & $13,00 \%$ & $27,60 \%$ \\
\hline & \multicolumn{3}{|c|}{ Observado } & \multicolumn{4}{|c|}{$\begin{array}{l}\text { Población negra pagada como grupo de refe- } \\
\text { rencia YB* - ecuación (4) }\end{array}$} \\
\hline & A & B & $\mathrm{C}$ & D & & & F \\
\hline & $\begin{array}{c}\text { Ingreso } \\
\text { otro grupo }\end{array}$ & $\begin{array}{c}\text { Ingreso } \\
\text { negra }\end{array}$ & (a)-(b) & $\mathrm{YB}^{*}$ & Delta & d)-(b) & $\begin{array}{l}\text { Efecto sobre } \\
\text { todo }(\mathrm{e}) /(\mathrm{c})\end{array}$ \\
\hline Mulata & 2,885 & 2,754 & $13,10 \%$ & 2,881 & & & $96,95 \%$ \\
\hline Mestiza & 3,147 & 2,754 & $39,30 \%$ & 3,042 & & & $73,28 \%$ \\
\hline Blanca & 3,225 & 2,754 & $47,10 \%$ & 3,0836 & & & $69,98 \%$ \\
\hline
\end{tabular}

Fuente: elaboración propia con base en la Encuesta Nacional de Hogares 2000 -etapa 110.

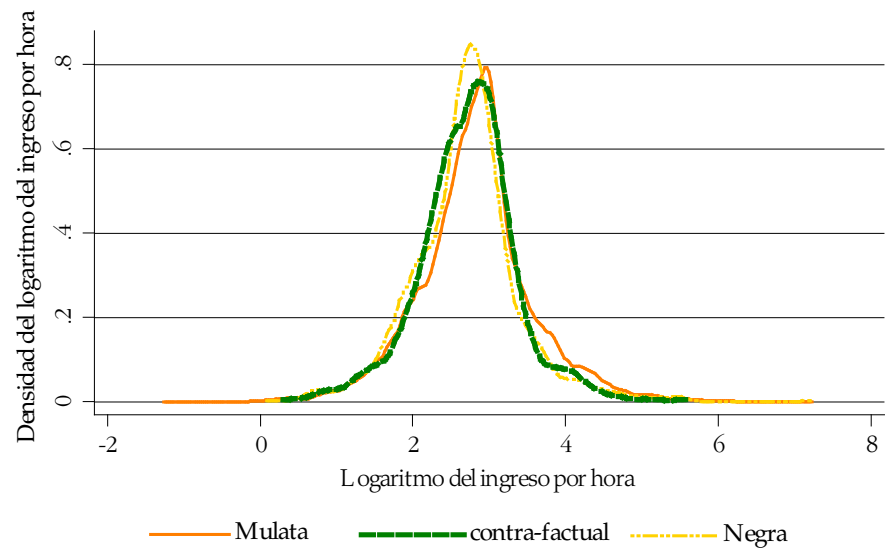

Figura 6. Función de densidad del logaritmo de ingresos Mulata frente a Negra ecuación 3

Fuente: elaboración propia con base en laEncuesta Nacional de Hogares 2000 -etapa 110. 


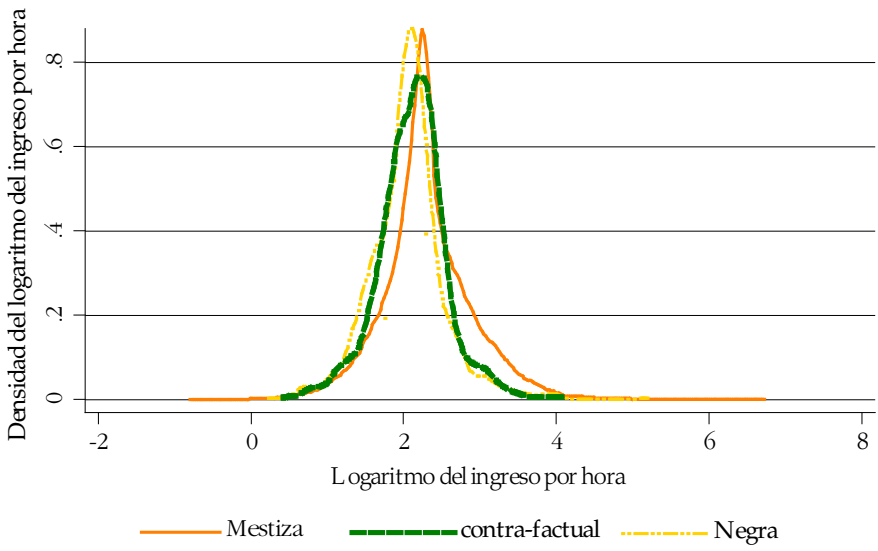

Figura 7. Función de densidad del logaritmo de ingresos Mestiza frente a Negra ecuación 3

Fuente: elaboración propia con base en la Encuesta Nacional de Hogares 2000 -etapa 110.

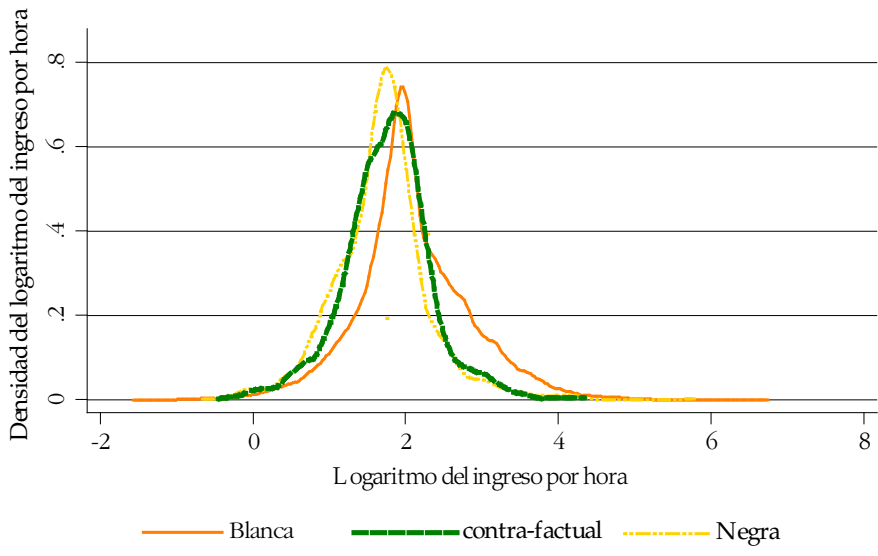

Figura 8. Función de densidad del logaritmo de ingresos Blanca frente a Negra ecuación 3

Fuente: elaboración propia con base en laEncuesta Nacional de Hogares 2000 -etapa 110. 


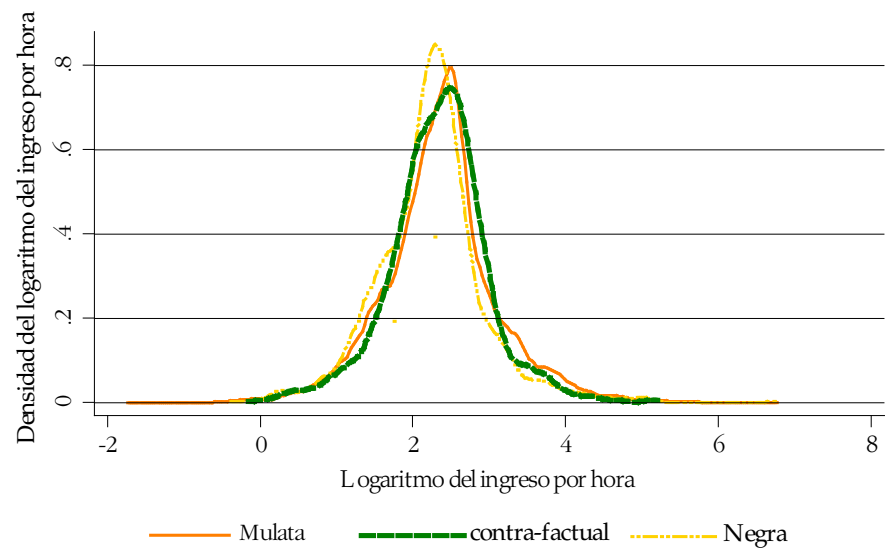

Figura 9. Función de densidad del logaritmo de ingresos Mulata frente a Negra ecuación 4

Fuente: elaboración propia con base en laEncuesta Nacional de Hogares 2000 -etapa 110.

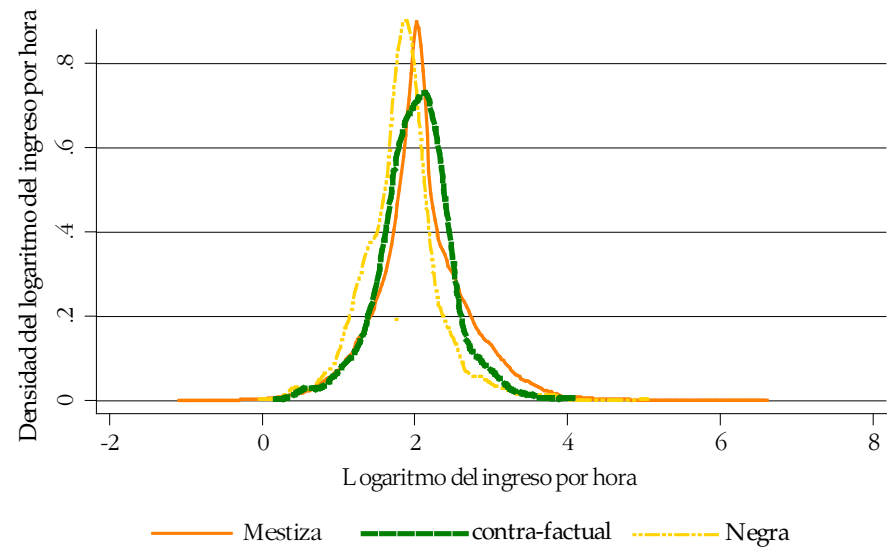

Figura 10. Función de densidad del logaritmo de ingresos Mestiza frente a Negra ecuación 4

Fuente: elaboración propia con base en laEncuesta Nacional de Hogares 2000 -etapa 110. 


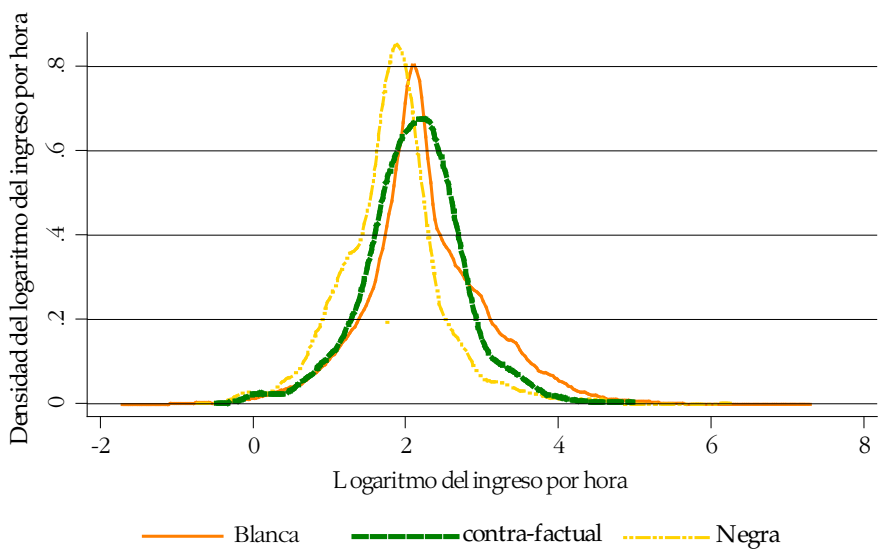

Figura 11. Función de densidad del logaritmo de ingresos Blanca frente a Negra ecuación 4

Fuente: elaboración propia con base en la Encuesta Nacional de Hogares 2000 -etapa 110.

\section{Consideraciones}

Los resultados revelan, en primer lugar, que existen brechas de ingreso importantes en Colombia para los grupos sociales diferenciados por el color de la piel. Esos diferenciales se incrementan monótonamente cuando se compara a personas con un color de piel más oscuro que el de otras personas. En segundo lugar, una vez se pagan a las personas negras con los retornos a los esfuerzos de los otros grupos, efecto de las circunstancias, las brechas de salarios se reducen de manera importante pero conservan el mismo comportamiento creciente y monótono a medida que el color de la piel se incrementa; el efecto precio es importante pero menos importante que el efecto de las circunstancias en los diferenciales de ingreso. En tercer lugar, cuando se mezclaron los efectos de las circunstancias y los precios o efecto global, se pudo corroborar que una proporción muy importante de los diferenciales de ingreso entre grupos sociales diferenciados por el color de la piel están asociados con la transmisión intergeneracional de las desigualdades por el color de la piel.

Los anteriores resultados revelan en su conjunto que se requiere mejorar los niveles de educación de la población negra, debido a que el efecto que mayor importancia tiene a la hora de determinar las brechas de ingreso son las características. Sin embargo, el trato desigual que recibe la población negra en el mercado laboral acrecienta las brechas, por lo que deben mejorarse 
las políticas y programas encargados de garantizar la no discriminación de ningún tipo en los lugares de trabajo.

\section{Referencias}

Andrews, D. W., Moreira, M. J., \& Stock, J. H. (2006). Optimal Two-Sided Invariant Similar Tests for Instrumental Variables Regression. Econometrica, 74(3), 715-752.

Andrews, G. R. (2016). Afro-Latin America: Black Lives, 1600-2000. Series:The Nathan I. Huggins Lectures 19. Cambridge: Harvard University Press.

Becker, G., \& Tomes, N. (1979). An Equilibrium Theory of the Distribution of Income and Intergenerational Mobility. Journal of Political Economy, 87(6), 1153-1189.

Behrman, J. R., Gaviria, A., \& Székely, M. (2001). Intergenerational mobility in Latin America. Fedesarrollo, Working paper 25.

Blinder, A. S. (1973). Wage Discrimination: Reduced Form and Structural Estimates. The Journal of Human Resources, 8(4), 436-455.

Bonilla, L. (2010). Movilidad intergeneracional en educación en las ciudades y regiones de Colombia. Revista de Economía del Rosario, 13(2), 191-233.

Bourguignon, F., Ferreira, F. H., \& Menéndez, M. (2003). Inequality of Outcomes and Inequality of Opportunities in Brazil. World Bank Policy Research, Working Paper 3174.

Bowles, S. (1973). Understanding Unequal Economic Opportunity. The American Economic Review, 63(2), 346-356.

Campante, F. R., Crespo, A. R., \& Leite, P. G. (2004). Desigualdade salarial entre raças no mercado de trabalho urbano brasileiro: aspectos regionais. Revista Brasileira de Economia, 58(2), 185-210.

Card, D. (1999). The causal effect of education on earnings. Handbook of labor economics, 3, 1801-1863.

Chevalier, A. (2004). Parental Education and Child's Education: A Natural Experiment. IZA Discussion Paper1153.

Dabady, M., Blank, R., \& Citro, C. (2004). Measuring Racial Discrimination. Washington D. C: National Academic Press.

Engerman, S. L., \& Sokoloff, K. L. (1997). Factor Endowments, Institutions, and Differential Growth Paths among New World Economies. En S. Haber, How Latin America Fell Behind (pp. 260-304). Stanford CA: Stanford University Press.

Ferreira, F. H., \& Meléndez, M. (2012). Desigualdad de resultados y oportunidades en Colombia: 1997-2010. Documentos CEDE 40. 
Flórez, C. E., Medina, C., \& Urrea, F. (2001). Understanding the cost of social exclusion due to race or ethnic background in Latin America and Caribbean Countries. Washington, DC: Inter-American Development Bank.

Fortin, N., Lemieux, T., \& Firpo, S. (2011). Decomposition Methods in Economics. En D. Card, \& O. Ashenfelte, Handbook of Labor Economics 4th Edition (pp. 1-102). Amsterdam: Elsevier North Holland.

Galvis-Aponte, L. A., \& Meisel-Roca, A. (2014). Aspectos regionales de la movilidad social y la igualdad. Revista de Economía del Rosario, 17(2), 257-297.

Gaviria, A. (2002). Los que suben y los que bajan. Educación y movilidad social en Colombia. Bogotá: Fedesarrollo.

Heckman, J. J. (1998). Detecting Discrimination. The Journal of Economic Perspective , 12(2), 101-116.

Leite, P. G. (2005). Race discrimination or inequality of opportunities: the Brazilian case. Discussion papers -Ibero America Institute for Economic Research 118.

Loury, G. C. (1981). Intergenerational Transfers and the Distribution of Earnings. Econometrica, 49(4), 843-867.

Nina, E., \& Grillo, S. (2000). Educación, movilidad social y "trampa de pobreza". Coyuntura Social Fedesarrollo22, 101-119.

Núñez, J., Ramírez, J. C., \& Taboada, B. (2006). Desigualdad de ingresos, esfuerzos y oportunidades - un estudio del caso colombiano. Documento Cede 11.

Oaxaca, R. (1973). Male-Female Wage Differentials in Urban Labor Markets. International Economic Review, 693-709.

Roemer, J. E. (1998). Equality of Opportunity. Cambidge: Harvard University, Press.

Ruiz, D. (2011). Desigualdad de oportunidades en Colombia. Documento de trabajo 142.

Sen, A. (1992). Inequality Reexamined. Cambridge, MA: Harvard University Press.

Soares, S. S. (2000). O perfil da discriminação no mercado de trabalho - homens negros, mulheres brancas e mulheres negras. IPEA Working Paper 769.

Tenjo, J. (2004). Educación y movilidad social en Colombia. Documentos de Economía 13.

Urrea, F., \& Viáfara, C. (2007). Pobreza y grupos étnicos en Colombia: Análisis de sus factores determinantes y lineamientos de politicas para su reducción. Bogotá: Departamento Nacional de Planeación.

Viáfara López, C. A. (2006). Efectos de la raza y el sexo en el logro educativo y en el estatus ocupacional en el primer empleo en la ciudad de Cali Colombia. Sociedad y Economía,11, 66-95. 
Viáfara López, C. A., Estacio Moreno, A., \& González Aguiar, L. M. (2010). Condición étnico-racial, género y movilidad social en Bogotá, Cali y el agregado de las trece áreas metropolitanas en Colombia: un análisis descriptivo y econométrico. Revista Sociedad y Economía,18, 113-136.

Viáfara, C. (2005). Diferencias raciales en las oportunidades educativas y ocupacionales en el primer empleo en la ciudad de Cali-Colombia. (Tesis de maestría, Flacso, México, D.F).

Viáfara, C., \& Urrea, F. (2006). Efectos de la raza y el género en el logro educativo y estatus socio-ocupacional para tres ciudades colombianas. Desarrollo y Sociedad,58, 115-163.

\section{Anexos}

\subsection{Anexo 1}

\section{ENCUESTA NACIONAL DE HOGARES}

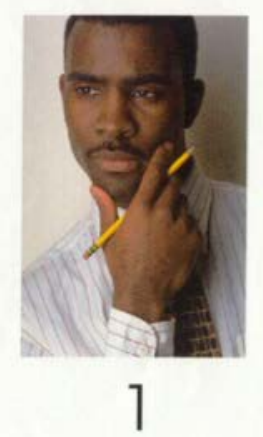

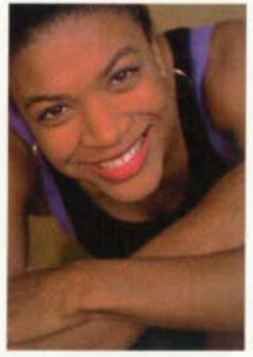

2

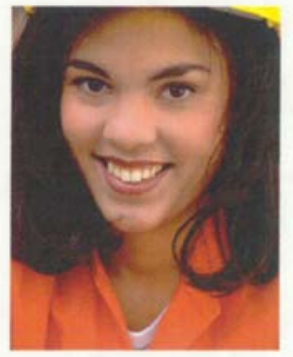

3

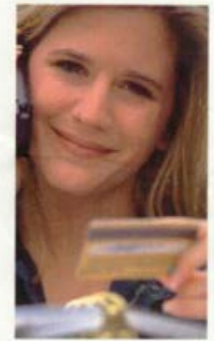

4 


\subsection{Anexo 2}

Anexo 2, Pruebas robustas sobre la debilidad del instrumento

Weak instrument robust tests for linear IV

HO: beta[lningrelah:anoeduca] $=0$

\begin{tabular}{|c|c|c|c|c|c|c|}
\hline Test & \multicolumn{3}{|c|}{ Statistic } & \multicolumn{3}{|c|}{ p-value } \\
\hline CLR & $\operatorname{stat}()$. & $=$ & 85.98 & Prob > stat & $=$ & 0.0000 \\
\hline $\mathrm{AR}$ & $\operatorname{chi} 2(15)$ & $=$ & 119.28 & Prob $>$ chi2 & $=$ & 0.0000 \\
\hline LM & $\operatorname{chi2}(1)$ & $=$ & 55.40 & Prob > chi2 & $=$ & 0.0000 \\
\hline$J$ & $\operatorname{chi2}(14)$ & $=$ & 63.88 & Prob $>$ chi2 & $=$ & 0.0000 \\
\hline LM-J & & B & rejecte & at 58 level & & \\
\hline Wald & $\operatorname{chi2}(1)$ & $=$ & 92.28 & Prob $>$ chi2 & $=$ & 0.0000 \\
\hline
\end{tabular}

Note: Wald test not robust to weak instruments.

Weak instrument robust tests for linear IV

HO: beta[lningrelah:anoeduca] $=0$

\begin{tabular}{|c|c|c|c|c|c|c|}
\hline Test & \multicolumn{3}{|c|}{ Statistic } & \multicolumn{3}{|c|}{ p-value } \\
\hline CLR & stat (.) & $=$ & 85.98 & Prob > stat & $=$ & 0.0000 \\
\hline $\mathrm{AR}$ & $\operatorname{chi2}(15)$ & $=$ & 119.28 & Prob $>$ chi2 & $=$ & 0.0000 \\
\hline LM & $\operatorname{chi2}(1)$ & $=$ & 55.40 & Prob > chi2 & $=$ & 0.0000 \\
\hline$J$ & $\operatorname{chi} 2(14)$ & $=$ & 63.88 & Prob $>$ chi2 & $=$ & 0.0000 \\
\hline LM-J & & B & rejecte & at 58 level & & \\
\hline Wald & $\operatorname{chi2}(1)$ & $=$ & 92.28 & Prob $>$ chi2 & $=$ & 0.0000 \\
\hline
\end{tabular}

Note: Wald test not robust to weak instruments. 